\title{
A Low-Carbon-Based Bilevel Optimization Model for Public Transit Network
}

\author{
Xu Sun, ${ }^{1}$ Hua-pu Lu, ${ }^{1}$ and Wen-jun $\mathrm{Chu}^{2}$ \\ ${ }^{1}$ Institute of Transportation Engineering, Tsinghua University, Beijing 100084, China \\ ${ }^{2}$ School of Traffic and Transportation, Beijing Jiaotong University, Beijing 100044, China \\ Correspondence should be addressed to Xu Sun; qingkong0113@126.com
}

Received 14 April 2013; Revised 6 June 2013; Accepted 6 June 2013

Academic Editor: Valentina E. Balas

Copyright (C) 2013 Xu Sun et al. This is an open access article distributed under the Creative Commons Attribution License, which permits unrestricted use, distribution, and reproduction in any medium, provided the original work is properly cited.

To satisfy the demand of low-carbon transportation, this paper studies the optimization of public transit network based on the concept of low carbon. Taking travel time, operation cost, energy consumption, pollutant emission, and traffic efficiency as the optimization objectives, a bilevel model is proposed in order to maximize the benefits of both travelers and operators and minimize the environmental cost. Then the model is solved with the differential evolution (DE) algorithm and applied to a real network of Baoji city. The results show that the model can not only ensure the benefits of travelers and operators, but can also reduce pollutant emission and energy consumption caused by the operations of buses, which reflects the concept of low carbon.

\section{Introduction}

With the rapid developments in urbanization and growth of car ownership, the pollution and energy consumption caused by traffic have become increasingly serious [1]. The low-carbon transportation system, which is characterized by low emission, low pollution, and low energy consumption, is an effective way to solve this problem [2-4]. Public transit, due to having a higher passenger capacity than cars [5], has been widely recognized as an important traffic mode in the low-carbon transportation system. The operation of transit vehicles can be affected by the transit network structures [6], so how to optimize the transit network is a key problem of the low-carbon system. In order to better meet the requirements of the low-carbon transport, it is necessary to optimize the transit network based on the concept of low carbon, which can make public transit become a more attractive travel mode by improving the transit service quality and reducing the operation cost.

In the past decades, several research efforts have examined the public transit network optimization problem and many optimization approaches have been proposed. Dubois et al. [7] designed transit network by identifying the roads needed for bus routes and choosing the set of bus routes. Then, frequencies of the designed routes were computed through a model aiming to minimize user waiting time. Hasselstrom [8] proposed a mathematical programming approach for transit network design by choosing the routes and determining frequencies concurrently. Ceder and Wilson [9] presented a new approach and an algorithm to design bus routes based on both passenger and operator interests. Baaj and Mahmassani [10] argued that a bus network could be generated by optimizing the route and the frequency, simultaneously. Van Nes et al. [11] presented a transit route design method, in which route or frequency optimization was based on an economic criterion. Pattnaik et al. [12] presented a genetic algorithm GA-based optimization method to design transit network. The objective of their optimization model was to minimize the total cost of user and operator. Agrawal and Mathew [13] presented an optimization model for transit network aiming to minimize the total system cost which is the sum of the operating cost and the generalized travel cost. Bielli et al. [14] developed a heuristic based on GA to design transit network to minimize the average travel time and management cost. Zhao [15] proposed a model for large-scale transit network aiming to minimize transfers and optimize the route/network directness. Yang et al. [16] proposed a mathematical model for transit network design aiming to maximize direct traveler density that meant the number of direct travelers carried by per unit length of a route. 
However, almost all the studies focused on the benefits of travelers and operators, meaning that the traditional way of optimizing transit network is to take the maximum benefits of travelers and operators as the optimization goal.

Considering the development trend of low-carbon transport, this paper attempts to combine the concept of lowcarbon transport with the traditional way of transit network optimization problem. To solve this problem, a new methodology is proposed in which the environment effect issue is explicitly considered in the process of network optimization for the first time. The proposed approach is presented using a bilevel optimization formulation. The outline of the paper is as follows: the next section describes the detailed optimization objectives and gives the representation of the branch objective functions. In the model description section, a bilevel optimization model is presented aiming to minimize the overall generalized cost of providing transit services. After that a solution algorithm is adopted for the bilevel programming approach based on the differential evolution (DE) algorithm. Numerical example section outlines the application of the proposed method to an example network. In the last section, the results are discussed and the major findings of this research are summarized.

\section{Transit Network Optimization Problem Statement}

2.1. The Basic Outline of Optimization Problem. The purpose of the low-carbon-based bilevel model is to determine a route network layout that minimizes the overall generalized cost of providing transit services, including the traveler cost, the operator cost, and the environmental cost. More specifically, the model will implement the objectives as follows:

(1) minimizing traveler cost: that is to reduce the total travel time and increase the rate of direct passengers by improving the density and service coverage of transit network,

(2) minimizing operator cost: that is to reduce the running cost by improving the operation efficiency and increase the profits of public transit enterprise by improving the transport capacity and the load factor,

(3) minimizing environmental cost: that is to reduce the pollutant emission and energy consumption by improving the operation efficiency and capacity of network.

2.2. Description of the Branch Objective Function. In this section, according to the three objectives mentioned above, some indicators including travel time, direct passengers, load factor, energy consumption, pollutant emission, and traffic efficiency are defined as the key branch objective functions of the optimization model to evaluate the impacts of the optimized transit network on the travelers, operators, and environment, respectively.

2.2.1. Representation of Traveler Cost Function. The impact on travelers refers to whether the optimized network can provide more convenient service for travelers by reducing the total travel time and transfer time. Therefore, the travel time and rate of direct passengers are used as the objective functions to represent the traveler cost.

Travel time:

$$
\begin{gathered}
f_{1}=\frac{\sum_{i, j \in N} q_{i j} t_{i j}}{\sum_{i, j \in N} q_{i j}}, \\
t_{i j}=\lambda_{1} t_{1}+\lambda_{2} t_{2}+\lambda_{3} t_{3}+\lambda_{4} t_{4} .
\end{gathered}
$$

Rate of direct passengers:

$$
f_{2}=\frac{\sum_{i, j \in N} V_{i j}}{\sum_{i, j \in N} q_{i j}} .
$$

2.2.2. Representation of Operator Cost Function. The impact on operators refers to whether the optimized network can increase the profits of enterprise and reduce the running cost of vehicles to minimize the operator cost. Therefore, the load factor and revenue rate are used to measure the operator cost.

Load factor:

$$
f_{3}=\frac{\sum_{k \in R} \sum_{i, j \in N} q_{i j, k} l_{i j k}}{\sum_{k \in R} \sum_{i, j \in N} Q_{i j, k} l_{i j k}} .
$$

Revenue rate:

$$
f_{4}=\frac{C_{1}}{C_{2}}=M+\frac{\sum_{i, j \in N} p_{i j} q_{i j}}{\sum_{k \in R} c_{r} q_{k} l_{k}+C_{s}+C_{v}} .
$$

2.2.3. Representation of Environmental Cost Function. The impact on environment refers to whether the optimized network can reduce the emission and energy consumption by improving the efficiency of network. Therefore, the pollutant emission, energy consumption as well as network efficiency are used to measure the generalized environmental cost.

Pollutant emission rate:

$$
f_{5}=\frac{\sum_{h \in H} \sum_{k \in R} \sum_{b \in B} l_{k} q_{k}^{b} \sigma_{h}^{b}\left(v_{k}^{b}\right)}{\sum_{h \in H} \sum_{k \in R} \sum_{b \in B} l_{k} q_{k}^{b} \sigma_{h s}^{b}\left(v_{k}^{b}\right)} .
$$

Energy consumption:

$$
f_{6}=\sum_{k \in R} \sum_{b \in B} \gamma l_{k} q_{k}^{b} \tau_{b}\left(v_{k}^{b}\right) .
$$

Network efficiency:

$$
f_{7}=\frac{\sum_{k \in R} \sum_{i, j \in N} q_{i j, k} \delta_{i j, k}}{\sum_{k \in R} l_{k}} .
$$

\section{Bilevel Model Formulation}

3.1. Upper-Level Formulation. The upper level model is articulated in accordance with the concept of low-carbon transport, the aim of which is the low emissions and low energy consumption caused by public transit. Therefore, the upper-level formulation is proposed in order to minimize 
the environmental cost. The objective function of upper-level model takes into account the emission, the energy consumption, and the operation efficiency, which can represent the environmental cost. According to formulations (6), (7), and (8), the objective function can be expressed as

$$
\min S(x)=w_{5} \alpha_{5} f_{5}+w_{6} \alpha_{6} f_{6}-w_{7} \alpha_{7} f_{7},
$$

where $w_{5}, w_{6}$, and $w_{7}$ are the weight coefficients and $\alpha_{5}, \alpha_{6}$, and $\alpha_{7}$ are transformation coefficients to convert the units of each term in the objective function (determined by AHP method).

In the upper-level model, the purpose of the objective function is to achieve the environment optima by minimizing the emission and energy consumption and maximizing the operation efficiency, which can meet the demand of lowcarbon transport.

3.2. Lower-Level Formulation. The lower-level model is formulated to optimize the transit network by minimizing both the traveler cost and operator cost. Therefore, the objective function of lower-level model is composed of the travel time and the direct passengers, which represent the traveler cost, as well as other evaluation indictors to represent the operator cost, including the load factor and revenue rate. According to formulations (1), (3), (4), and (5), the objective function of lower-level model is expressed as

$$
\min Z(x)=w_{1} \alpha_{1} f_{1}-w_{2} \alpha_{2} f_{2}-w_{3} \alpha_{3} f_{3}-w_{4} \alpha_{4} f_{4},
$$

where $w_{1}, w_{2}, w_{3}$, and $w_{4}$ are the weight coefficients and $\alpha_{1}$, $\alpha_{2}, \alpha_{3}$, and $\alpha_{4}$ are the transformation coefficients.

In the lower-level model, the purpose of the objective function is to achieve the optima for both travelers and operators by minimizing the travel time and maximizing the rate of direct passengers, load factor, and revenue rate.

\section{Solution Algorithm}

4.1. Differential Evolution Algorithm. The differential evolution (DE) algorithm was first proposed by Storn and Price [17]. As a stochastic and parallel searched algorithm, the $\mathrm{DE}$ algorithm has been demonstrated to be an effective and robust method for global optimization.

The DE algorithm is a population-based algorithm, which combines simple arithmetic operators with the classical events of crossover, mutation, and selection to evolve from randomly generated initial population to final individual solution [18]. In detail, the mutation and crossover operators are used to generate the trial vectors, and selection is then used to determine whether the new generated vectors can survive the next generation. Because it has simple structure and local searching property and requires few control parameters, fast convergence, the DE algorithm is regarded as one of the best evolutionary algorithms and widely used to solve optimization problems.

According to some studies [19-21], DE algorithm can obtain a better solution and has the better performance than other population-based evolutionary algorithms when applied to solve diverse combinatorial optimization problems with continuous variables. So this paper attempts to use DE algorithm to solve the bilevel optimization model.

4.2. Application of DE Algorithm to Bilevel Model. The bilevel model even with linear objective functions and constraints at both levels is an NP-hard problem and difficult to solve. Moreover, there are many variables in the model proposed in this paper, and the solution domain and objective function vary with the change of feature vectors; the traditional deterministic methods cannot guarantee the global optimum. So the DE algorithm, due to its global search capability independent of gradient information, is applied to solve this bilevel optimization problem. The detailed DE algorithm can be described as follows.

(i) Parameters Initialization. The main parameters of DE algorithm are population size $N$, length of the chromosome $D$, the mutation factor $F$, the crossover rate $C R$, and the maximum generations number $G$. The mutation factor $F$ is selected in $[0,2]$; the crossover rate CR is selected in $[0,1]$.

(ii) Population Initialization. The initial population is randomly generated within the boundary using the following formulation:

$$
x_{i j}^{0}=x_{j}^{\min }+\operatorname{rand} \times\left(x_{j}^{\max }-x_{j}^{\min }\right),
$$

where $i=1,2, \ldots, N, j=1,2, \ldots, D, x_{j}^{\min }$ and $x_{j}^{\max }$ are the minimum and maximum limits of $j$ th dimension, respectively, and rand denotes a uniform random number between $[0,1]$.

(iii) Mutation. The mutation operation creates a new vector by adding the weighted difference of two random vectors to a third vector. For each vector $x_{i}^{G}$ in generation $G$, the mutant vector $v_{i}^{G+1}$ is created according to the following equation:

$$
v_{i}^{G+1}=x_{r_{1}}^{G}+F\left(x_{r_{2}}^{G}-x_{r_{3}}^{G}\right) \text {, }
$$

where $F$ is a mutation factor used to control the amplification of the differential variation; $G$ is the current generation number; and $r_{1}, r_{2}$, and $r_{3}$ are three distinct random numbers and none of them coincides with the current target number $i\left(r_{1} \neq r_{2} \neq r_{3} \neq i\right)$.

(iv) Crossover. Crossover operation can increase the diversity of the population. The trail vector $u_{i}^{G+1}$ is generated by mixing the mutated vectors $v_{i}^{G+1}$ with the target vectors $x_{i}^{G}$ according to the following rules:

$$
u_{i j}^{G+1}= \begin{cases}v_{i j}^{G+1} & \text { if } \operatorname{rand}(j) \leq \mathrm{CR}, j=\operatorname{rand} n(t), \\ x_{i j}^{G} & \text { otherwise, }\end{cases}
$$

where rand $(j) \in[0,1]$ is a randomly generated number with uniform distributed; $j$ represents the $j$ th dimension; and rand $n(t) \in[1,2, \ldots, D]$ is a randomly selected integer to ensure that the trail vector gets at least one parameter from mutated vector. 


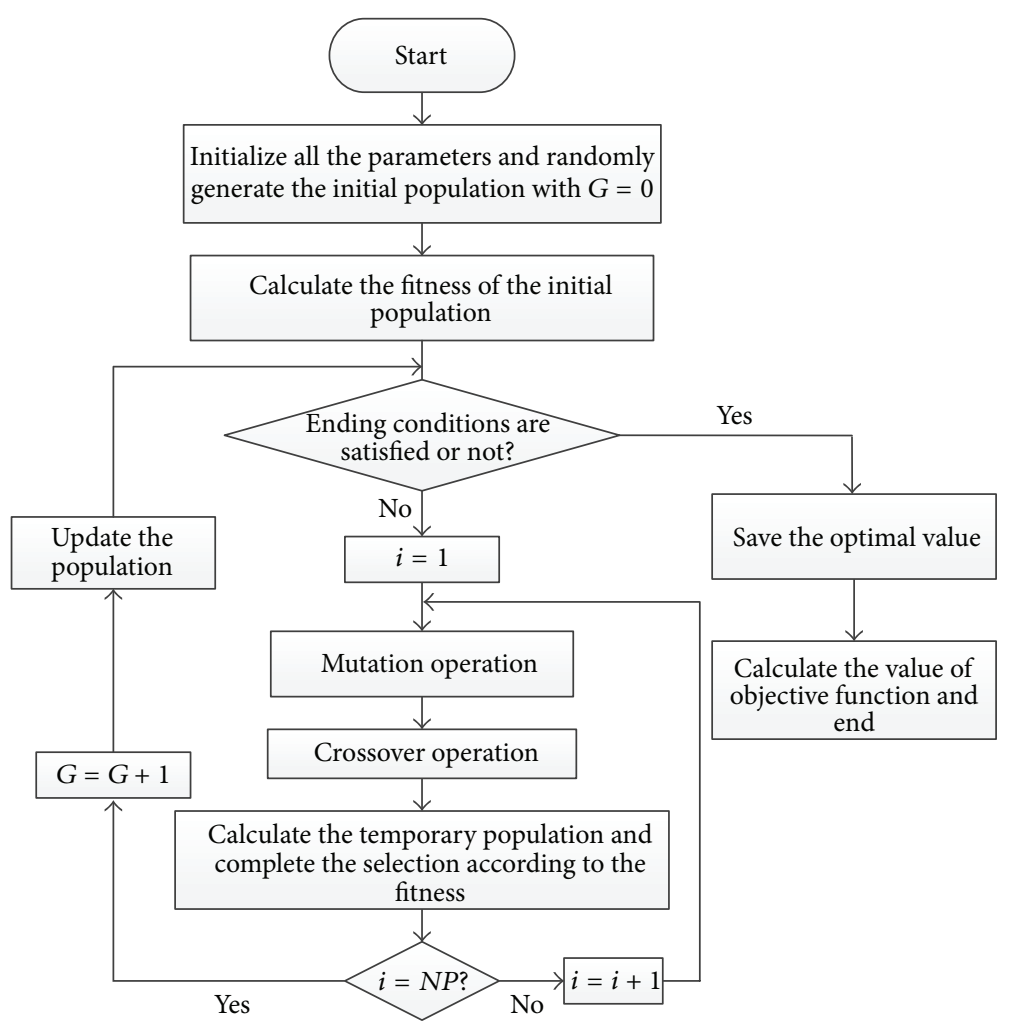

FIGURE 1: The flowchart of the DE-based solution approach.

(v) Selection. Selection operation retains the better offspring in the next generation. The generated offspring $u_{i}^{G+1}$ replaces the parent $x_{i}^{G}$, only if the fitness of the offspring $f\left(u_{i}^{G+1}\right)$ is better than that of the parent $f\left(x_{i}^{G}\right)$ :

$$
x_{i}^{G+1}= \begin{cases}u_{i}^{G+1} & \text { if } f\left(u_{i}^{G+1}\right) \leq f\left(x_{i}^{G}\right), \\ x_{i}^{G} & \text { otherwise. }\end{cases}
$$

(vi) The Determination of Weight Coefficient. The weight coefficient of objective function is determined by the entropyweight method, which is described as

$$
\begin{gathered}
H_{u}=(-\ln n)^{-1} \sum_{e=1}^{n} p_{u e} \ln p_{u e}, \\
w_{u}=1-\frac{H_{u}}{\sum_{u=1}^{m}\left(1-H_{u}\right)},
\end{gathered}
$$

where $w_{u}$ is the weight coefficient of the $u$ th indicator $f_{u}$ of objective function and $\sum_{u=1}^{m} w_{u}=1 ; H_{u}$ is the entropy values; and $p_{u e}=r_{u e} / \sum_{e=1}^{n} r_{u e}, r_{u e} \in[0,1]$.

(vii) The Calculation of Object Function. The value of the objective function can be calculated as

$$
f(x)=\sum_{u=1}^{m} w_{u} f_{u}
$$

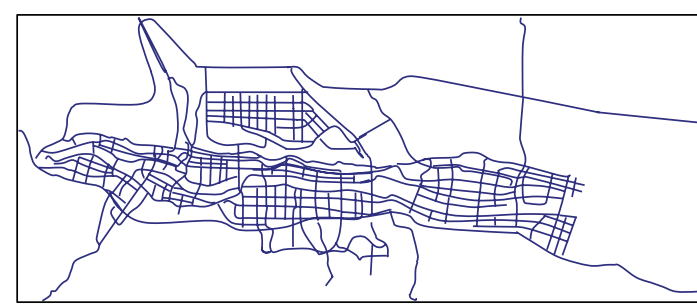

FIgURE 2: The urban transit network of Baoji.

In this bilevel model, the optimization problem of upper level model is defined as min $f(x)$ and can be solved with the algorithm mentioned previously, which is also applicable for the lower level subproblem. The flowchart of the DE-based solution approach is illustrated in Figure 1.

\section{Numerical Example}

In this section, the proposed model and method are applied to a real transit network in Baoji city, China. Figure 2 shows the layout of the network. There are 38 bus routes and 418 bus stops, which extends $463.65 \mathrm{~km}$, and 865 buses carrying 184.53 million passengers a year. The other detailed data used in this example, such as the passenger stop OD matrix, the density of transit network, and the nonlinear coefficient of bus route, can be obtained from our former research and found in [22]. 
TABLE 1: Comparison of the optimization model results with the existing transit network.

\begin{tabular}{lccccccc}
\hline Indicators & $f_{1} / \mathrm{min}$ & $f_{2} / \%$ & $f_{3} / \%$ & $f_{4} / \%$ & $f_{5} / \%$ & $f_{6} / 10^{4}$ ton & $f_{7} / \%$ \\
\hline Existing results & 38.7 & 66.3 & 54.4 & 83.6 & 133.4 & 1.58 & 59.7 \\
Optimal results & 33.5 & 71.5 & 60.3 & 87.5 & 121.3 & 1.49 & 64.8 \\
Ratio enhancement & $-13.4 \%$ & $7.2 \%$ & $10.8 \%$ & $4.6 \%$ & $-9.1 \%$ & $-4.4 \%$ & $8.5 \%$ \\
\hline
\end{tabular}

The solution process is as follows.

Step 1 (determining the weight coefficient). According to formula (15), the weight coefficients of the indicators in the model can be calculated as

$$
\begin{gathered}
w_{1}=0.3124, \quad w_{2}=0.2072, \\
w_{3}=0.1849, \quad w_{4}=0.2955, \\
w_{5}=0.3451, \quad w_{6}=0.3382, \quad w_{7}=0.3167 .
\end{gathered}
$$

Step 2 (determining the transformation coefficient). Correspondingly, the transformation coefficients of these indicators can be determined by using the analytic hierarchy process:

$$
\begin{gathered}
\alpha_{1}=0.2254, \quad \alpha_{2}=0.2846, \\
\alpha_{3}=0.3011, \quad \alpha_{4}=0.1889, \\
\alpha_{5}=0.2813, \quad \alpha_{6}=0.2951, \quad \alpha_{7}=0.4236 .
\end{gathered}
$$

Step 3 (parameters calibration). The parameters used in the DE algorithm are defined: population size $N=40$, mutation factor $F=1.3$, crossover rate $\mathrm{CR}=0.8$, and maximum generations number $G=200$.

Step 4 (implementing the DE algorithm). The DE algorithm procedure, which is proposed for solving the bilevel model of transit network optimization, is coded by MATLAB 2009 and implemented on a computer with a $2.2 \mathrm{GHz}$ CPU. Table 1 presents the optimal results of evaluation indicators, which are calculated from the optimization model. For comparison, the existing results of corresponding indicators are also included, which are the real data obtained from the traffic survey.

According to Table 1, the following can be clearly seen.

(i) For the travelers, the average travel time decreases by $5.2 \mathrm{~min}$ and the rate of direct passengers increases by $7.2 \%$, which indicates that the optimized transit network becomes more convenient for travelers by improving density and service coverage of the network and ensures the maximum benefits of travelers.

(ii) For the operators, there is an improvement of $10.8 \%$ for the average load factor of network and $4.6 \%$ for revenue rate of transit enterprise, indicating that the optimized network enables improving the operator benefits by increasing transport capacity and operation efficiency.

(iii) For the environment, the amounts of pollutant emission and energy consumption decrease by $9.1 \%$ and $4.4 \%$, respectively, while the operation efficiency of network increases by $8.5 \%$. It shows that the optimized network achieves the goal of low emission, low energy consumption, and high efficiency.

\section{Conclusions}

The transit network optimization problem is an extremely complex problem with multi objectives and constraints. This paper combined the concept of low-carbon transportation into the transit network optimization problem, which means that the environment effect should be considered in the process of network optimization. A low-carbon-based bilevel optimization model was proposed aiming to minimize the overall generalized cost of providing transit services, including the traveler cost, the operator cost, and the environmental cost. Then the model was solved with the DE algorithm and applied to a real network of Baoji city. The application results showed that the optimization model can not only make the transit network more convenient and efficient by improving the direct passengers and service coverage, but can also ensure the environmental benefits in terms of lower energy consumption, pollution, and emission.

\section{Notation}

$f_{1}$ : $\quad$ Average travel time of travelers

$f_{2}$ : Rate of direct passengers

$f_{3}$ : Load factor of the network

$f_{4}$ : Revenue rate of operators

$f_{5}$ : Pollutant emissions rate

$f_{6}$ : Energy consumption

$f_{7}$ : Network efficiency

$i, j$ : Index of node in the transit network

$k$ : Index of transit route

$b$ : Index of vehicle type

$h$ : Index of pollutant type, $h=1,2,3,4$

represents $\mathrm{CO}, \mathrm{CO}_{2}, \mathrm{NO}_{x}, \mathrm{HC}$,

respectively

$N$ : $\quad$ Set of nodes in the transit network

$R: \quad$ Set of transit routes

$B$ : $\quad$ Set of vehicle types

$H$ : Set of pollutant types

$q_{i j}$ : Number of trips originating from node $i$ and destined for node $j$

$q_{i j, k}$ : Number of trips from $i$ to $j$ on route $k$

$Q_{i j, k}$ : Vehicle seating capacity from $i$ to $j$ on route $k$

$V_{i j}$ : Number of direct trips originating from node $i$ and destined for node $j$ 


\begin{tabular}{|c|c|}
\hline$t_{i j}:$ & Total travel time between nodes $i$ and $j$ \\
\hline$t_{1}:$ & Average walking time \\
\hline$t_{2}:$ & Average waiting time \\
\hline$t_{3}:$ & Average transfer time \\
\hline$t_{4}:$ & Average in-vehicle travel time \\
\hline$\lambda_{i}(i=1,2,3,4)$ & $\begin{array}{l}\text { Adjusted coefficient (can be determined } \\
\text { by Delphi method) }\end{array}$ \\
\hline$l_{k}:$ & Length of route $k$ \\
\hline$l_{i j k}:$ & $\begin{array}{l}\text { Length from } i \text { to } j \text { measured along the } \\
\text { route } k\end{array}$ \\
\hline$\delta_{i j k}:$ & $\begin{array}{l}\text { Percentage of the number of trips from } i \\
\text { to } j \text { distributed on route } k\end{array}$ \\
\hline$C_{1}:$ & $\begin{array}{l}\text { Total revenue of public transit } \\
\text { enterprise }\end{array}$ \\
\hline$C_{2}:$ & The total cost \\
\hline$M:$ & Subsidies provided by the government \\
\hline$p_{i j}:$ & Ticket price from $i$ to $j$ \\
\hline$c_{r}:$ & Per-kilometer operating cost of a bus \\
\hline$C_{s}:$ & Acquisition cost of transit vehicles \\
\hline$C_{v}:$ & Maintenance cost of transit vehicles \\
\hline$q_{k}:$ & Number of operating buses on route $k$ \\
\hline$q_{k}^{b}:$ & $\begin{array}{l}\text { Number of transit vehicles of type } b \text { on } \\
\text { route } k\end{array}$ \\
\hline$v_{k}^{b}:$ & $\begin{array}{l}\text { Average speed of transit vehicles of type } \\
b \text { on route } k\end{array}$ \\
\hline$\sigma_{h}^{b}\left(v_{k}^{b}\right):$ & $\begin{array}{l}\text { Actual concentration of pollutant } h \text { at } \\
\text { the speed of } v_{k}^{b} \text { for vehicle } b\end{array}$ \\
\hline$\sigma_{h s}^{b}\left(v_{k}^{b}\right):$ & $\begin{array}{l}\text { Standard concentration of pollutant } h \text { at } \\
\text { the speed of } v_{k}^{b} \text { for vehicle } b\end{array}$ \\
\hline$\tau_{b}\left(v_{k}^{b}\right)$ & $\begin{array}{l}\text { Energy consumption factor at the speed } \\
\text { of } v_{k}^{b} \text { for vehicle } b \text { on the route } k\end{array}$ \\
\hline & Conversion coefficient of energy. \\
\hline
\end{tabular}

\section{Acknowledgments}

The authors are grateful to the editor and anonymous reviewers for their valuable suggestions which improved the paper. This work is partly supported by Science and Technology Program of Beijing, China (Grant no. Z121100000312101).

\section{References}

[1] L. Zhang, "The research on low-carbon transport situation and countermeasure in China," Energy Conservation Technology, vol. 3, no. 1, pp. 79-83, 2013.

[2] M. Meng, C. F. Shao, and X. Zhang, "Research of traffic network equilibrium model with low carbon emissions constraints," Disaster Advances, vol. 5, no. 4, pp. 713-716, 2012.

[3] M. Su, R. Li, W. Lu, C. Chen, B. Chen, and Z. Yang, "Evaluation of a low-carbon city: method and application," Entropy, vol. 15, no. 4, pp. 1171-1185, 2013.

[4] T. Zhang, "Research on urban low-carbon transport development index," Technology Economics, vol. 32, no. 3, pp. 78-84, 2013.

[5] S. M. Feng and H. R. Chen, "Study of public transit network optimization method," Journal of Harbin Institute of Technology, vol. 37, no. 5, pp. 691-693, 2005.
[6] Y. Zhao and S. Zhong, "Optimization for the urban transit routing problem based on the genetic algorithm," Computer Engineering and Science, vol. 34, no. 4, pp. 109-112, 2013.

[7] D. Dubois, G. Bel, and M. Llibre, "A set of methods in transportation network synthesis and analysis," Journal of the Operational Research Society, vol. 30, no. 9, pp. 797-808, 1979.

[8] D. Hasselstrom, Public transportation planning-a mathematical programming approach [Ph.D. thesis], University of Goteborg, Goteborg, Sweden, 1981.

[9] A. Ceder and N. H. M. Wilson, "Bus network design," Transportation Research Part B, vol. 20, no. 4, pp. 331-344, 1986.

[10] M. H. Baaj and H. S. Mahmassani, "Hybrid route generation heuristic algorithm for the design of transit networks," Transportation Research Part C, vol. 3, no. 1, pp. 31-50, 1995.

[11] R. van Nes, R. Hamerslag, and B. H. Immerse, "Design of public transportation networks," Transportation Research Record, vol. 1202, pp. 74-82, 1998.

[12] S. B. Pattnaik, S. Mohan, and V. M. Tom, "Urban bus transit route network design using genetic algorithm," Journal of Transportation Engineering, vol. 124, no. 4, pp. 368-375, 1998.

[13] J. Agrawal and T. V. Mathew, "Transit route network design using parallel genetic algorithm," Journal of Computing in Civil Engineering, vol. 18, no. 3, pp. 248-256, 2004.

[14] M. Bielli, M. Caramia, and P. Carotenuto, "Genetic algorithms in bus network optimization," Transportation Research $C$, vol. 10, no. 1, pp. 19-34, 2002.

[15] F. Zhao, "Transit network optimization-minimizing transfers and optimizing route directness," Journal of Public Transportation, vol. 7, no. 1, pp. 63-82, 2004.

[16] Z. Yang, B. Yu, and C. Cheng, "A parallel ant colony algorithm for bus network optimization," Computer-Aided Civil and Infrastructure Engineering, vol. 22, no. 1, pp. 44-55, 2007.

[17] R. Storn and K. Price, "Differential evolution: a simple and efficient heuristic for global optimization over continuous spaces," Journal of Global Optimization, vol. 11, no. 4, pp. 341359, 1997.

[18] L. Wang, C.-X. Dun, W.-J. Bi, and Y.-R. Zeng, "An effective and efficient differential evolution algorithm for the integrated stochastic joint replenishment and delivery model," KnowledgeBased Systems, vol. 36, pp. 104-114, 2012.

[19] Q. Feng and D. Y. Zhou, "Time optimal path planning based on different evaluation algorithm," Computer Engineering and Application, vol. 31, no. 12, pp. 74-76, 2005.

[20] J. J. Wang, L. Li, D. Niu, and Z. Tan, "An annual load forecasting model based on support vector regression with differential evolution algorithm," Applied Energy, vol. 94, pp. 65-70, 2012.

[21] S. A. Taher and S. A. Afsari, "Optimal location and sizing of UPQC in distribution networks using differential evolution algorithm," Mathematical Problems in Engineering, vol. 2012, Article ID 838629, 20 pages, 2012.

[22] H. P. Lu et al., The Comprehensive Transport Planning for Baoji City, Institute of Transportation Engineering, Tsinghua university, Beijing, China, 2011. 


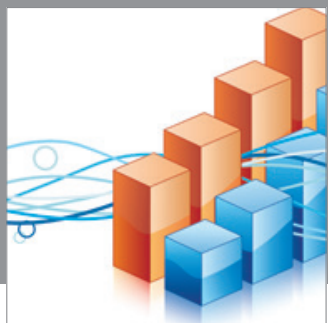

Advances in

Operations Research

mansans

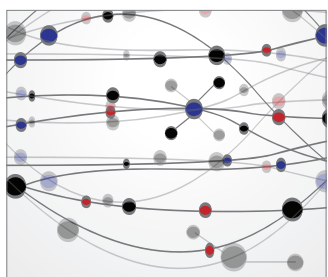

The Scientific World Journal
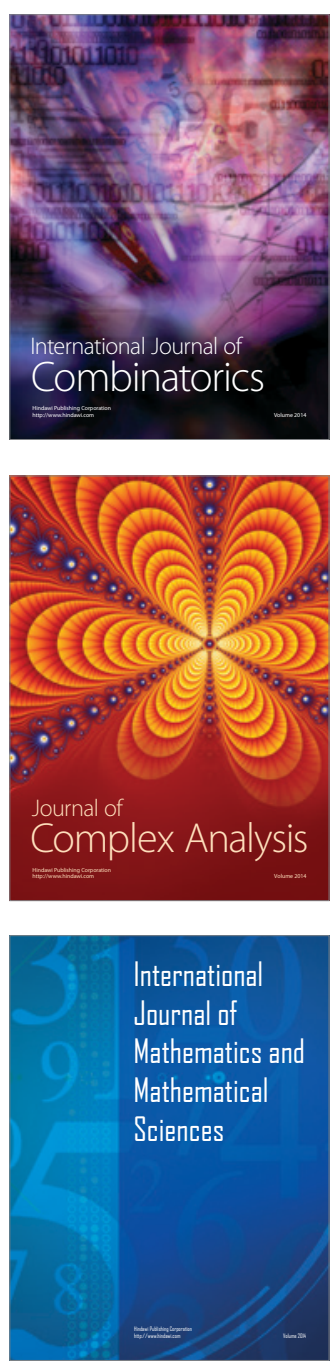
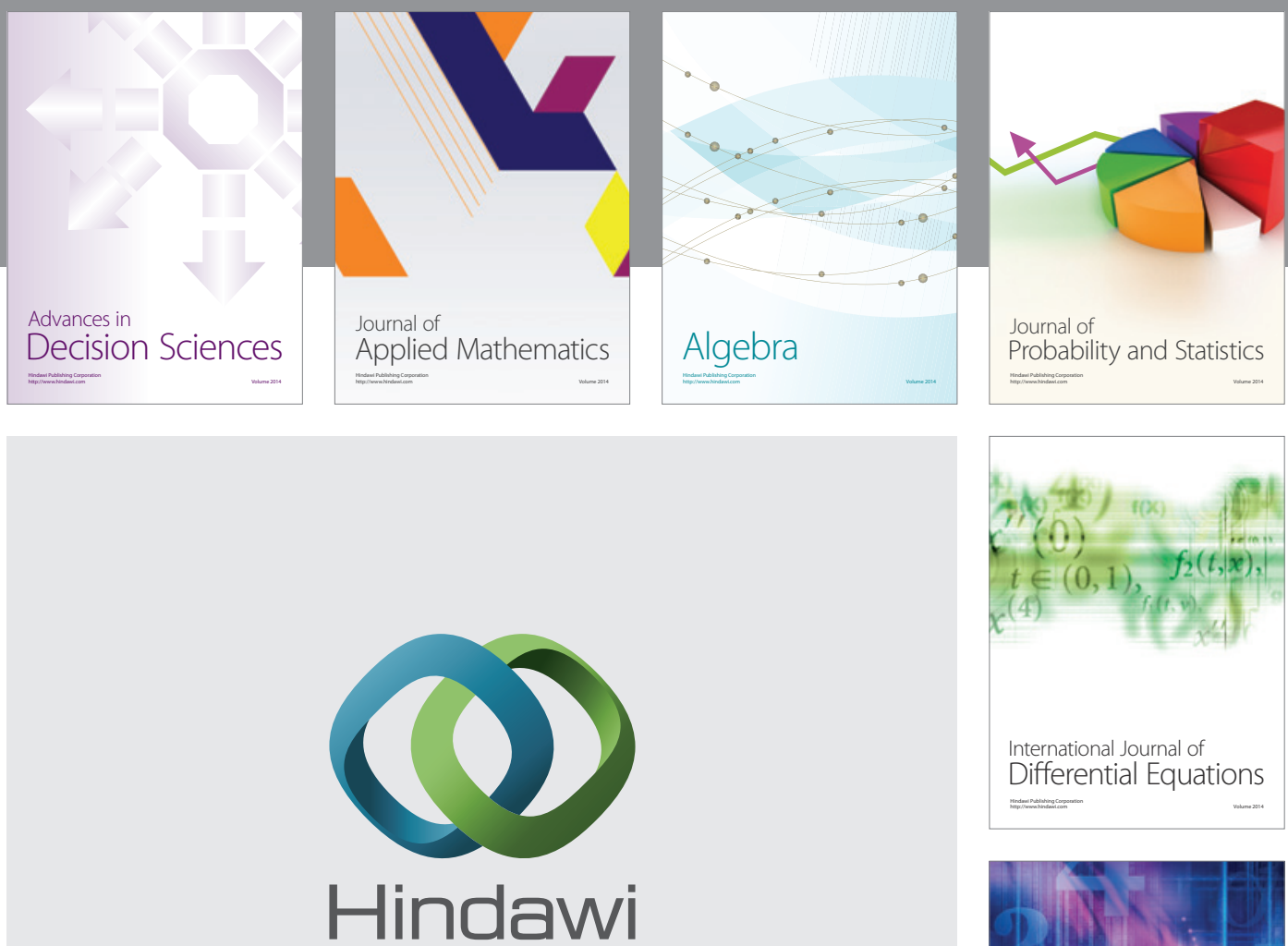

Submit your manuscripts at http://www.hindawi.com
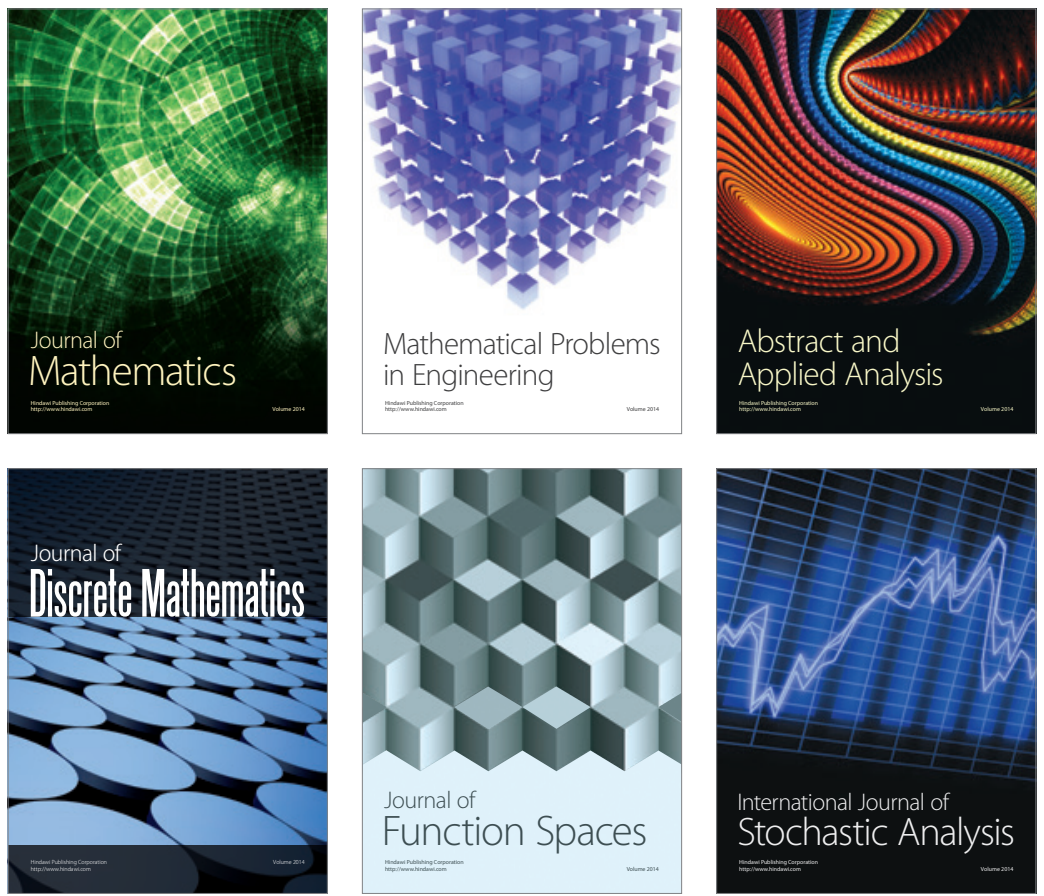

Journal of

Function Spaces

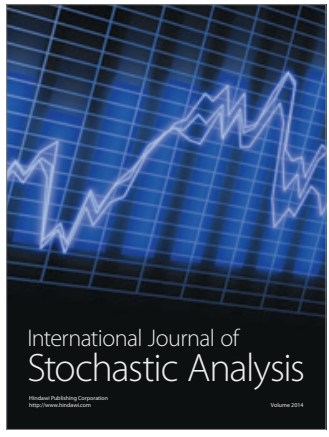

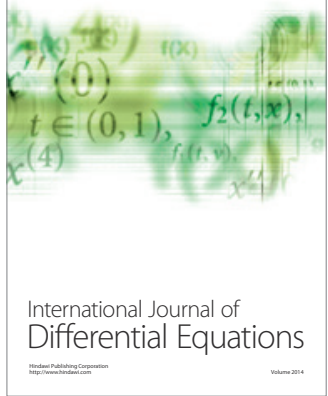
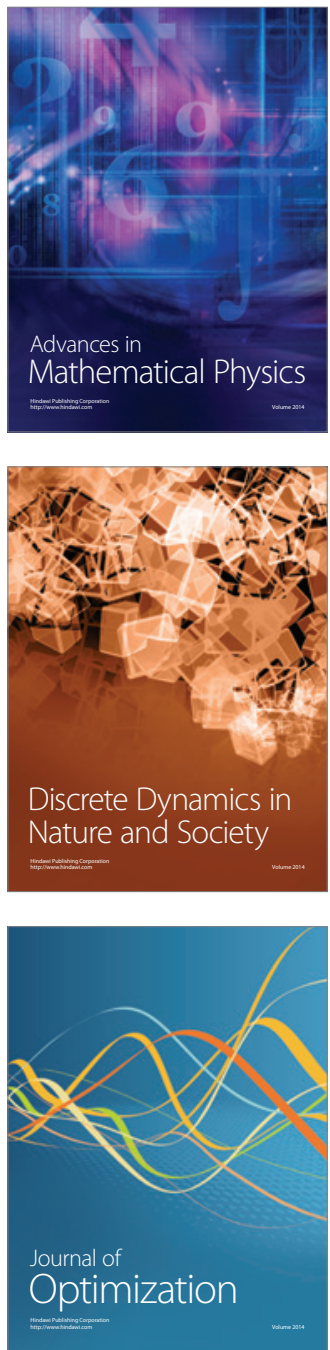